\section{EMBRYRIDDLE \\ Aeronautical University}

SCHOLARLY COMMONS
International Journal of Aviation, Aeronautics, and Aerospace

\title{
A New Method for the Prediction of the Downwash Angle Gradient
}

MONDHER YAHYAOUI Dr.

Ecole d'aviation de Borj El Amri, Tunisia., mondher_yahia@yahoo.fr

Follow this and additional works at: https://commons.erau.edu/ijaaa

Part of the Aerodynamics and Fluid Mechanics Commons

\section{Scholarly Commons Citation}

YAHYAOUI, M. (2019). A New Method for the Prediction of the Downwash Angle Gradient. International Journal of Aviation, Aeronautics, and Aerospace, 6(3). https://doi.org/10.15394/ijaaa.2019.1336

This Article is brought to you for free and open access by the Journals at Scholarly Commons. It has been accepted for inclusion in International Journal of Aviation, Aeronautics, and Aerospace by an authorized administrator of Scholarly Commons. For more information, please contact commons@erau.edu. 
A New Method for the Prediction of the Downwash Angle Gradient

Cover Page Footnote

None

This article is available in International Journal of Aviation, Aeronautics, and Aerospace: https://commons.erau.edu/ ijaaa/vol6/iss3/9 


\section{Introduction}

The downwash angle gradient is an important parameter in the analysis of the aerodynamics and longitudinal stability of airplanes. It affects mainly the aircraft overall lift-curve slope and the position of the neutral point position which in turn defines the longitudinal static stability, control, and maneuverability.

The overall lift coefficient of an airplane is given by $C_{L}=a \alpha$ with (Etkin \& Reid, 1996):

$$
a=a_{w b}+\eta_{H} \frac{S_{H}}{S}\left(1-\frac{\partial \varepsilon}{\partial \alpha}\right) a_{H}
$$

where $a_{w b}$ is the lift-curve slope for the wing-body combination and $\eta_{H}$ is the ratio of the dynamic pressure acting on the horizontal tail to that relative to the wings.

In steady flight, the pitching moment coefficient is given by:

$$
C_{m}=C_{m_{0}}+C_{m_{\alpha}} \alpha+C_{m_{\delta_{e}}} \delta_{e}
$$

Where $\delta_{e}$ is the elevator deflection angle, and:

$$
C_{m_{0}}=C_{m_{a c_{w b}}}+\eta_{H} V_{H} a_{H}\left(i_{H}+\varepsilon_{0}\right)\left[1-\eta_{H} \frac{a_{H}}{a} \frac{S_{H}}{S}\left(1-\frac{\partial \varepsilon}{\partial \alpha}\right)\right]
$$

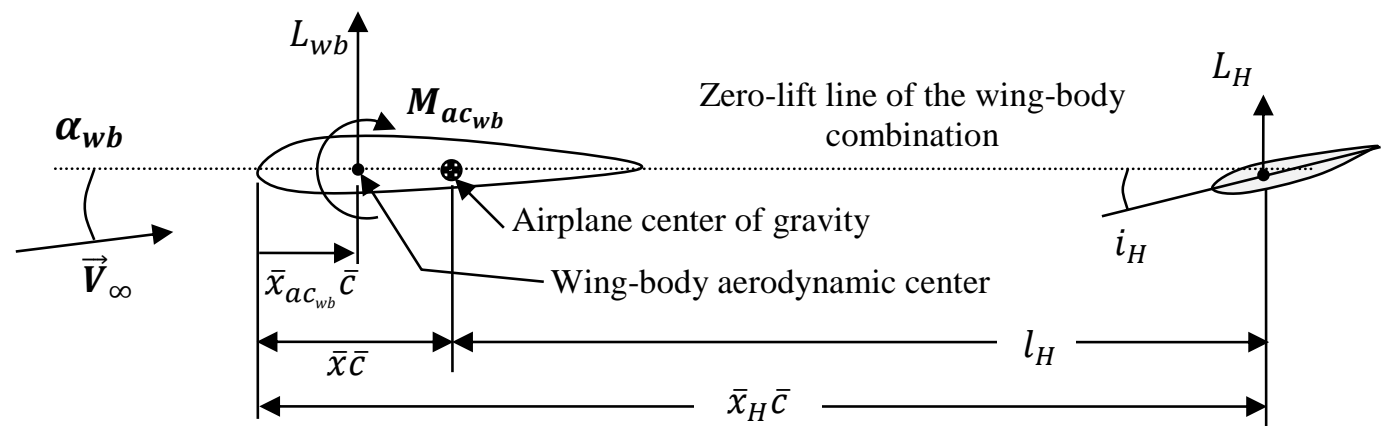

Figure 1. Parameters related to aircraft longitudinal stability.

In equation (2):

$$
\begin{aligned}
& C_{m_{\alpha}}=-a\left(\bar{x}_{n}-\bar{x}\right) \\
& \bar{x}_{n}=\frac{\bar{x}_{a c_{w b}}+\left(a / a_{w b}-1\right) \bar{x}_{H}}{a / a_{w b}} \\
& \frac{a}{a_{w b}}=1+\eta_{H} \frac{S_{H}}{S} \frac{a_{H}}{a_{w b}}\left(1-\frac{\partial \varepsilon}{\partial \alpha}\right)
\end{aligned}
$$


Equations (1)-(5) show the importance of the downwash angle gradient $\partial \varepsilon / \partial \alpha$.

The downwash field has been the subject of several research efforts reaching as far back as the late thirties of the twentieth century (Silverstein \& Katzoff, 1939; Silverstein, Katzoff, \& Bullivant, 1939). In fact, the only scarce data of practical use in the preliminary phases of aircraft design (Raymer, 2012; Roskam, 1979), available in the academic world, is derived from the experimental data (Silverstein \& Katzoff, 1939). This data is given in the form of charts for the downwash angle gradient at the centerline for unswept wings with aspect ratios of 6,9 , and 12 , and taper ratios of $1,0.33$, and 0.2 . In addition to the fact that no data is available for swept wings, the range of values is rather limited in terms of both aspect and taper ratio. Interpolation for other aspect and taper ratios is likely to introduce some additional error. In particular, if linear interpolation is used, the additional error will be somewhat limited when interpolating for taper but more important when interpolating for aspect ratio. This can be seen from Figure 2 where the variation of the downwash angle gradient with taper is close to linear but its variation with aspect ratio suggests that a higher order interpolation should be used.

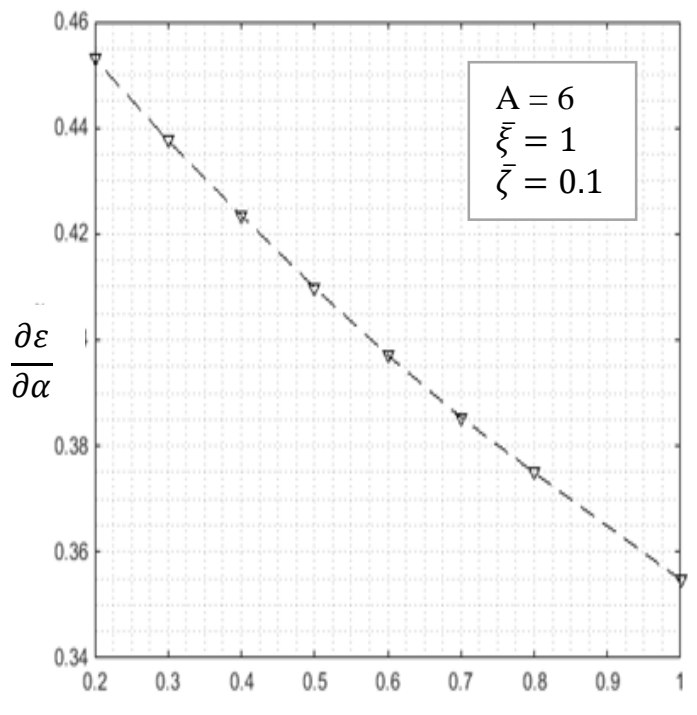

(a)

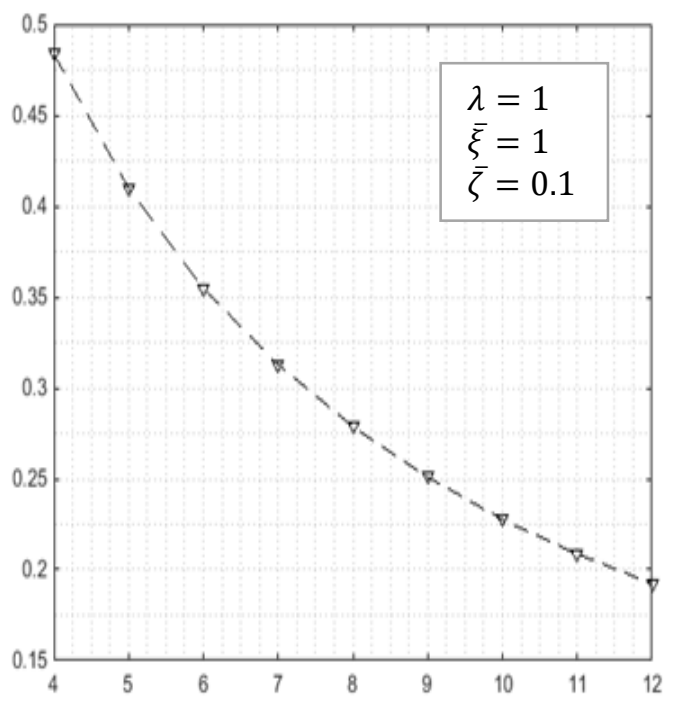

(b)

Figure 2. Variation of $\partial \varepsilon / \partial \alpha$ (a) with taper for $A=6$ and (b) with aspect ratio for a taper ratio $\lambda=1$ (VLM computations).

On the other hand, the widely referenced empirical law from the USAFDATCOM (Hoak et al., 1978) was found by Levy (1992) and in the present work to often enough underestimate the downwash angle gradient. This is shown in Figure 3 where, for some cases, the discrepancy with regard to experimental values is not negligible. It can be as high as $27 \%$. Since the charts (Raymer, 2012; Roskam, 
1979) give the downwash angle gradient on the centerline, the values were multiplied by a correction factor (Roskam, 1979) to get the average gradient across the horizontal tail span.
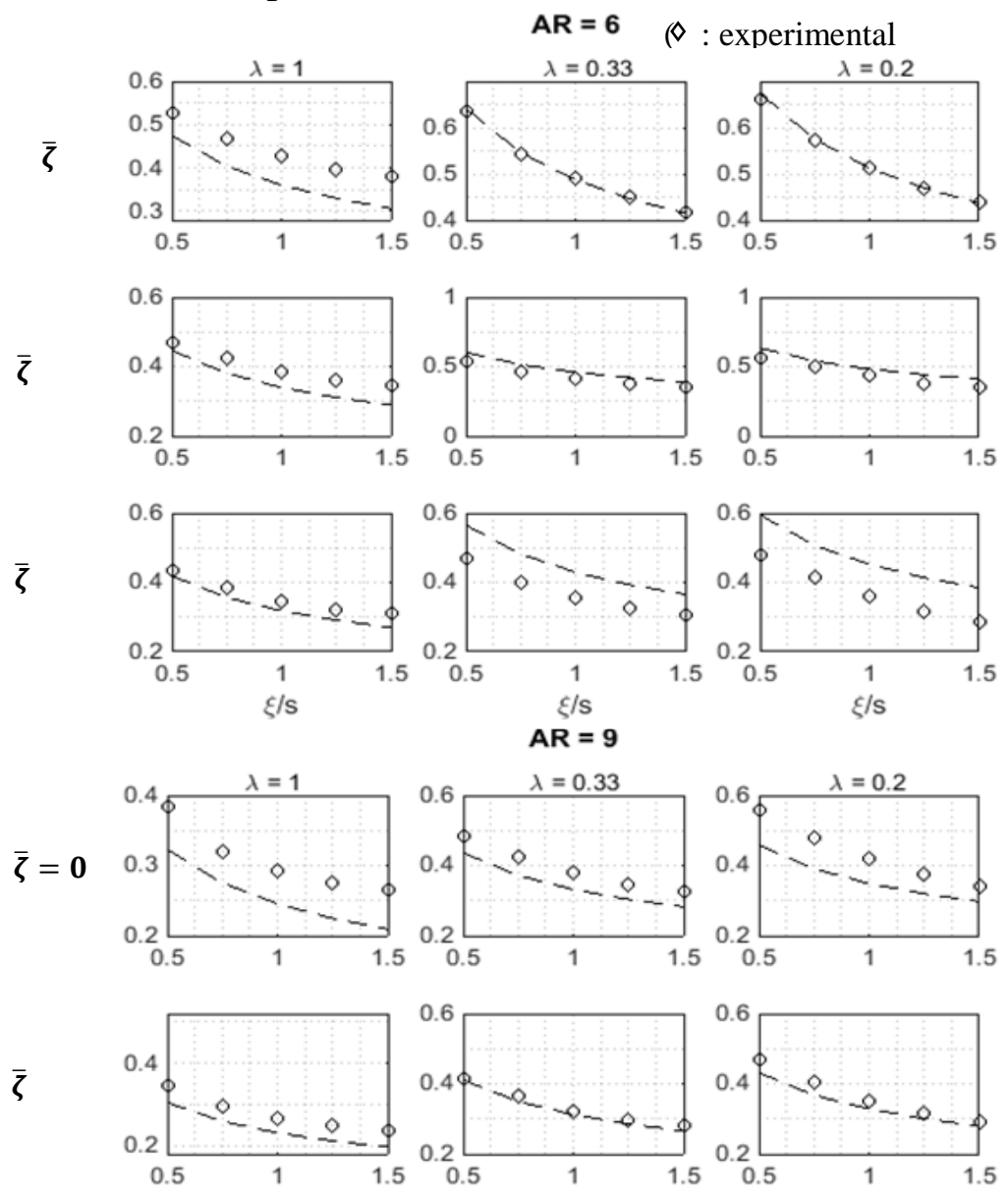

$\bar{\zeta}$
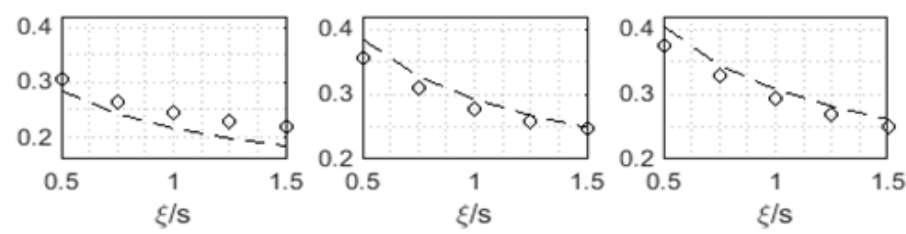

Figure 3. Comparison of USAF-DATCOM empirical law with experimental values of the average downwash angle gradient $\partial \varepsilon / \partial \alpha$ (Raymer, 2012; Roskam, 1979). 
In Figure 3, the parameters $\xi$ and $\zeta$ are the coordinates of the horizontal tail aerodynamic center defined on Figure 4.

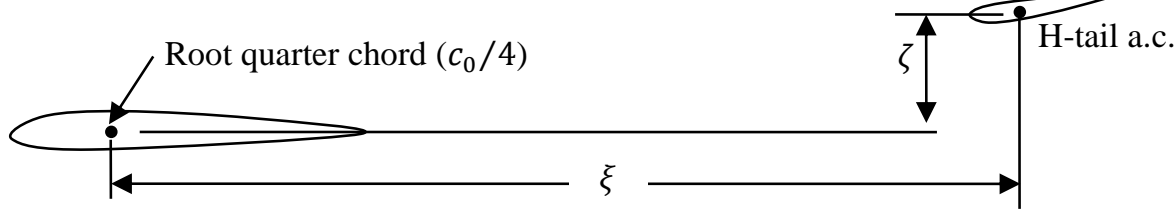

Figure 4. Geometric parameters for horizontal tail location.

The previous considerations set the premise for the present study where the downwash angle gradient is accurately estimated for values of aspect ratio ranging from 4 to 12 and values of taper ratio going from 0.2 to 1 .

\section{The Curve Fit Approach}

For straight and tapered but unswept wings, the new method was first introduced as a mere curve fit to experimental or numerical data of the form:

$$
\frac{\partial \varepsilon}{\partial \alpha}=\frac{1+c_{1} \bar{\xi}}{c_{2}+c_{3} \bar{\xi}}
$$

where $\bar{\xi}=\xi / s, s$ being the wing half span. The three unknown constants $c_{1}, c_{2}$, and $c_{3}$ in equation (7) were determined by solving a linear system of equations obtained by requiring that the curve representing the downwash angle gradient go through three arbitrary points of coordinates $\bar{\xi}_{1}, \bar{\xi}_{2}$ and $\bar{\xi}_{3}$. It was found that choosing $\bar{\xi}_{1}$ to be the abscissa of the first data element, $\bar{\xi}_{3}$ as that of the last data element and $\bar{\xi}_{2}$ as the abscissa of the middle element of the data set yields curves which fairly accurately match the experimental data. The three unknown constants are thereby solutions to the following linear system:

$$
\left(\begin{array}{lll}
\bar{\xi}_{1} & -f\left(\bar{\xi}_{1}\right) & -\bar{\xi}_{1} f\left(\bar{\xi}_{1}\right) \\
\bar{\xi}_{2} & -f\left(\bar{\xi}_{2}\right) & -\bar{\xi}_{2} f\left(\bar{\xi}_{2}\right) \\
\bar{\xi}_{3} & -f\left(\bar{\xi}_{3}\right) & -\bar{\xi}_{3} f\left(\bar{\xi}_{3}\right)
\end{array}\right)\left[\begin{array}{l}
c_{1} \\
c_{2} \\
c_{3}
\end{array}\right]=\left[\begin{array}{l}
-1 \\
-1 \\
-1
\end{array}\right]
$$

where $f=\partial \varepsilon / \partial \alpha$.

\section{Application to Experimental Data}

The three constants needed to implement equation (7) have been computed for all cases given in the charts (Raymer, 2012; Roskam, 1979) which give the downwash angle gradient at the centerline $\left(\varepsilon_{c}\right)$ and not its average over the horizontal tail span. 
First, for each pair of values of $A$ and $\lambda$, five experimental values of $\partial \varepsilon_{c} / \partial \alpha$ were accurately read from the charts using plot digitizing software at the five streamwise locations $\bar{\xi}=0.5,0.75,1,1.25$, and 1.5 . Then the linear system (8) was set up using the three collocation points $\bar{\xi}=0.5,1$, and 1.5 . It was then solved for the constants $c_{1}, c_{2}$, and $c_{3}$. The values are given in Table 1 .

Table 1

Constants $c_{1}, c_{2}$, and $c_{3}$ for the Experimental Data (Raymer, 2012; Roskam, 1979)

\begin{tabular}{|c|c|c|c|c|c|c|c|c|c|c|}
\hline \multicolumn{3}{c|}{} & \multicolumn{3}{c|}{$\mathbf{A = 6}$} & \multicolumn{3}{c|}{$\mathbf{A = 9}$} & \multicolumn{3}{c|}{$\mathbf{A = 1 2}$} \\
\hline$\lambda$ & $\zeta / s$ & $c_{1}$ & $c_{2}$ & $c_{3}$ & $c_{1}$ & $c_{2}$ & $c_{3}$ & $c_{1}$ & $c_{2}$ & $c_{3}$ \\
\hline \multirow{3}{*}{1} & 0 & 0.80 & 1.14 & 3.31 & 2.99 & -0.63 & 14.95 & 4.00 & -2.78 & 27.78 \\
\cline { 2 - 12 } & 0.1 & 0.84 & 1.34 & 3.71 & 1.30 & 0.95 & 8.09 & 1.91 & -0.36 & 16.37 \\
\cline { 2 - 12 } & 0.2 & 1.02 & 1.21 & 4.94 & 1.45 & 1.29 & 9.29 & 2.23 & -0.74 & 20.19 \\
\hline \multirow{3}{*}{0.33} & 0 & 0.31 & 0.88 & 1.53 & 0.46 & 1.13 & 2.43 & 0.37 & 1.65 & 2.36 \\
\cline { 2 - 11 } & 0.1 & 0.33 & 1.01 & 1.90 & 0.59 & 1.19 & 3.35 & 1.60 & 1.03 & 8.02 \\
\cline { 2 - 11 } & 0.2 & 0.55 & 0.97 & 2.97 & 0.98 & 1.16 & 5.41 & 2.94 & -0.19 & 16.74 \\
\hline \multirow{3}{*}{0.2} & 0 & 0.33 & 0.83 & 1.44 & 0.15 & 0.99 & 1.48 & 0.09 & 1.49 & 1.30 \\
\cline { 2 - 10 } & 0.1 & -0.05 & 1.09 & 0.85 & 0.13 & 1.21 & 1.67 & -0.06 & 1.76 & 1.27 \\
\cline { 2 - 10 } & 0.2 & 0.00 & 1.22 & 1.23 & 0.29 & 1.55 & 2.41 & 0.27 & 1.97 & 3.19 \\
\hline
\end{tabular}

Figure 5 shows how the above values of constants $c_{1}, c_{2}$, and $c_{3}$ and equation (7) allow for a thorough reproduction of the experimental values.
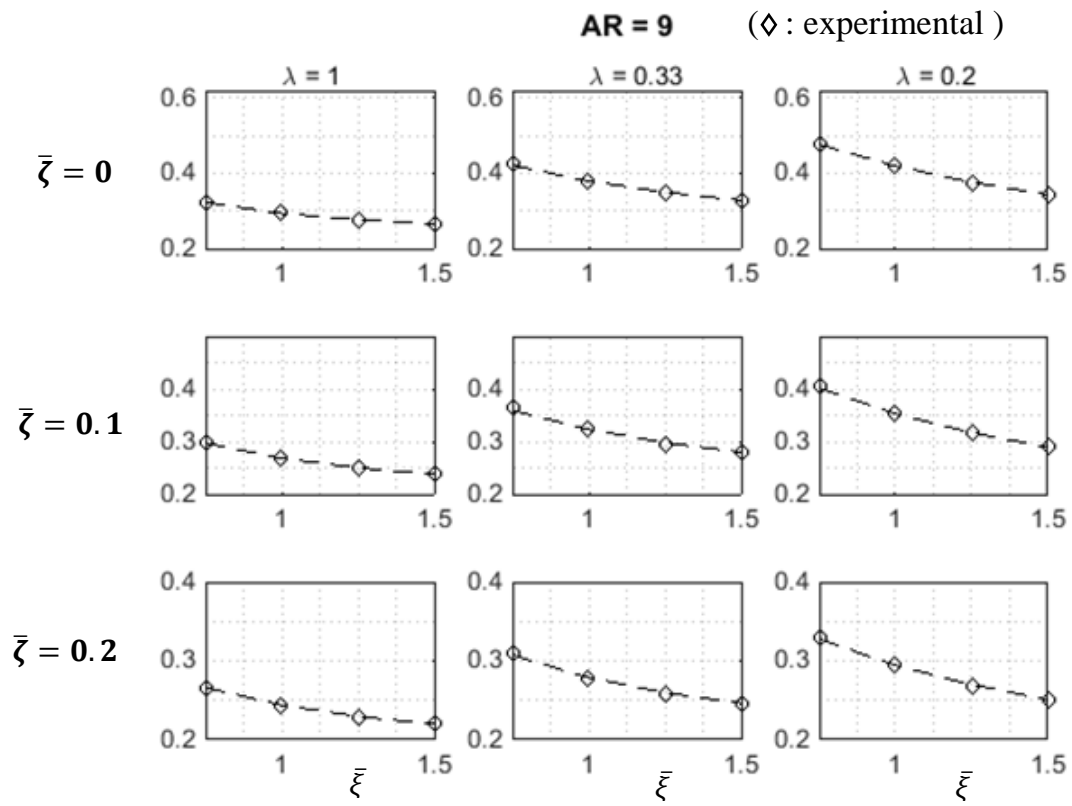

Figure 5. Example of agreement between the present curve-fit approach and experimental values of $\partial \varepsilon_{c} / \partial \alpha$ (Roskam, 1979; Raymer, 2012). 
More results for straight as well as swept wings will be obtained using the VLM for a wider range of aspect and taper ratios.

\section{Application to Numerical Values}

\section{Validation of the VLM computations}

The vortex-lattice method used in this investigation is a singularity method which has been around for many decades and is well documented in the literature (Bertin \& Smith, 1998). A VLM code has been developed in the MATLAB environment and used to compute the downwash field aft of wings of arbitrary configurations.

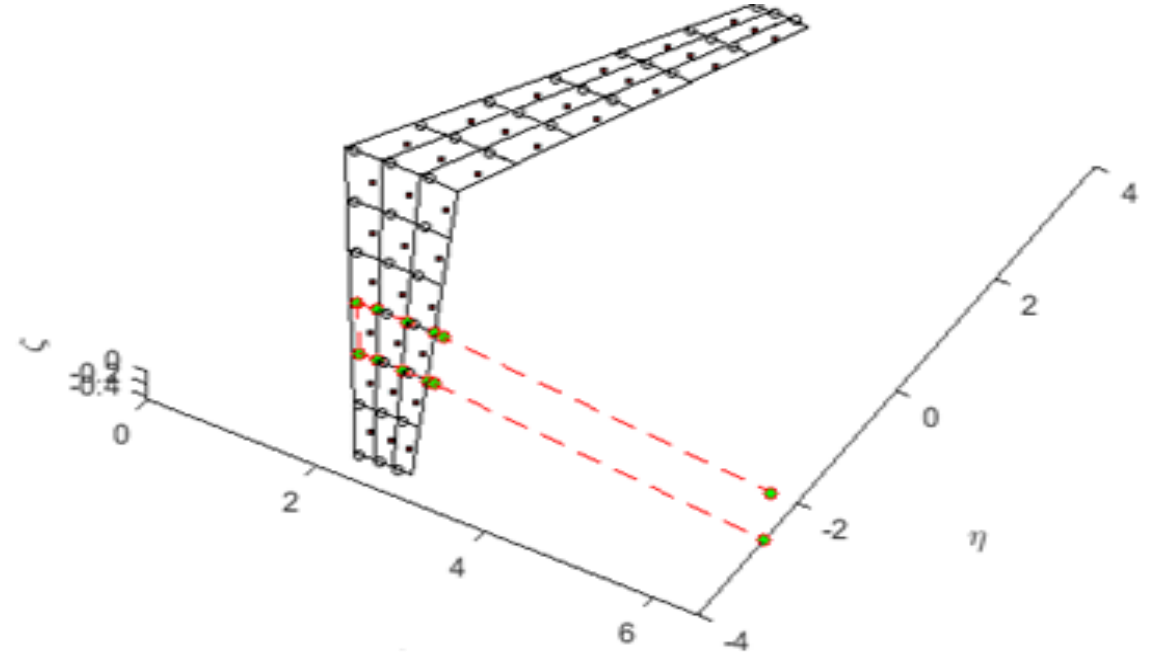

Figure 6. Example of a swept and tapered wing treated by the MATLAB code.

A lattice resolution of three chordwise rows and 24 rows per semi span was sufficiently accurate. Experimenting with the wake geometry, it was found that the choice which gave the closest agreement with experimental data was that of trailing vortex legs leaving the trailing edge as straight segments extending to infinity parallel to the local chord.

In order to validate our VLM computations, a first comparison is made with experimental values of $\partial \varepsilon_{c} / \partial \alpha$ (Raymer, 2012; Roskam, 1979). Sample results are given in Figure 7 for an aspect ratio of 9. Computations were also carried out for aspect ratios of 6 and 12 . The agreement is quite satisfactory since the average relative difference between the VLM results and the experimental values at a typical distance aft of the wing quarter center chord $\bar{\xi}=1$ is around $3 \%$ for all three aspect and taper ratios at $\bar{\zeta}=0 \bar{\zeta}=0.1$, and $\bar{\zeta}=0.2$. If each aspect ratio is looked at separately, the difference is equal to $3.5 \%$ for aspect ratios of 6 and 12 only about $2 \%$ for an aspect ratio of 9 . 


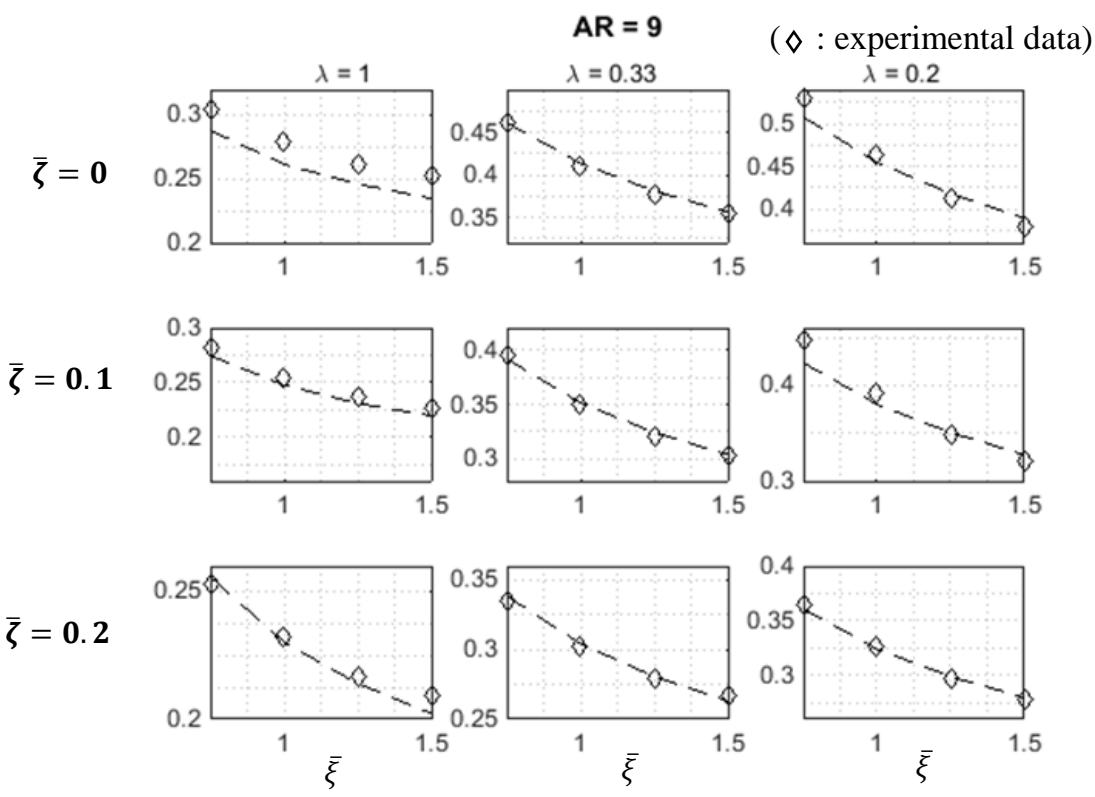

Figure 7. Comparison of VLM and experimental values of $\partial \varepsilon_{c} / \partial \alpha$ (Raymer, 2012).

A second comparison is made with experimental values of the downwash angle at the center line (Silverstein, Katzoff, \& Bullivant, 1939). As shown on Figure 8 , the agreement is quite good including the case of a $20^{\circ}$ flap deflection, especially at typical downstream locations of the horizontal tail $(\bar{\xi}=1)$.

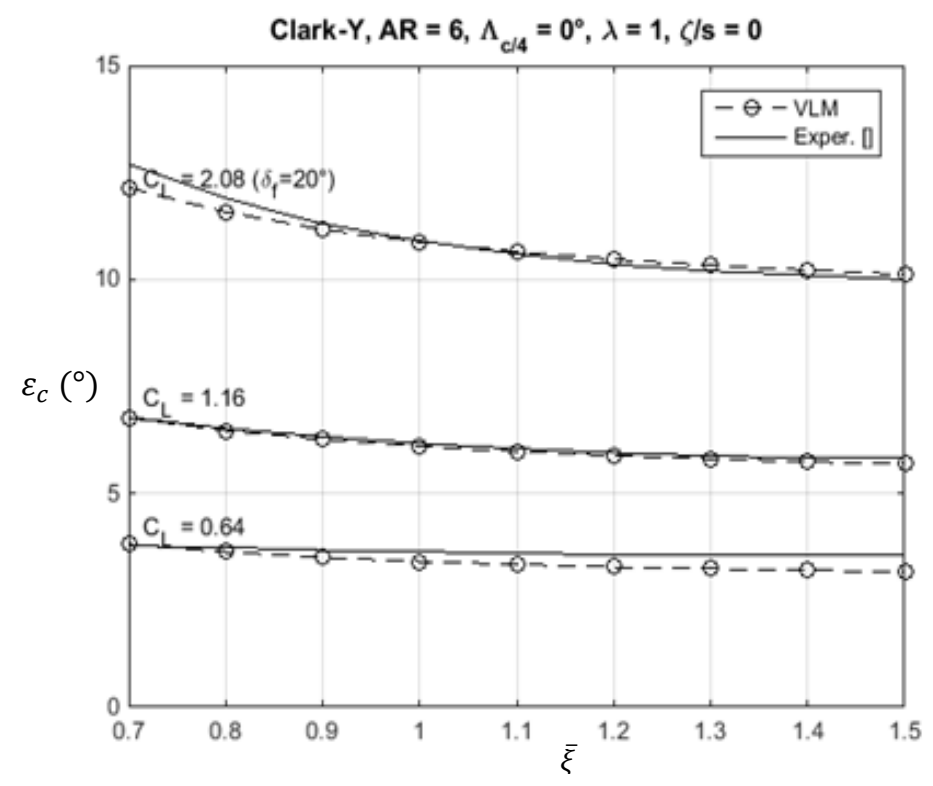


Figure 8. Comparison of downwash angle values at the plane of symmetry given by the VLM and experimental ones (Silverstein, Katzoff, \& Bullivant, 1939).

The average relative difference is less than $2.5 \%$ for a $\bar{\xi}$ between 0.7 and 1 and reaches $4.8 \%$ at $\bar{\xi}=1.5$.

\section{New Results Using the VLM}

Since we have shown that the VLM results are accurate enough to be used in conceptual and preliminary design studies, more data for the downwash angle gradient can now be generated. Values of the three constants needed to evaluate the average downwash angle gradient for straight, tapered, and untwisted wings in the incompressible regime are given in Table 2 for values of aspect ratio ranging from 4 to 12 and values of taper ratio going from 0.2 to 1 . These constants are based on average values of the downwash angle gradient over the span of the tail, which is equal to $40 \%$ of the wing's span.

Table 2

Constants $c_{1}, c_{2}$, and $c_{3}$ for Straight Tapered Wings (VLM computations)

\begin{tabular}{|c|c|c|c|c|c|c|c|c|c|c|}
\hline & \multicolumn{3}{|c|}{$A=4$} & \multicolumn{3}{|c|}{$A=5$} & \multicolumn{3}{|c|}{$A=6$} \\
\hline$\lambda$ & $\bar{\zeta}$ & $c_{1}$ & $c_{2}$ & $c_{3}$ & $c_{1}$ & $c_{2}$ & $c_{3}$ & $c_{1}$ & $c_{2}$ & $c_{3}$ \\
\hline \multirow{3}{*}{1} & 0 & 2.73 & -0.30 & 7.38 & 2.42 & -0.12 & 7.81 & 2.38 & -0.08 & 8.89 \\
\hline & 0.1 & 1.84 & 0.06 & 5.81 & 1.75 & 0.16 & 6.55 & 1.76 & 0.18 & 7.60 \\
\hline & 0.2 & 0.98 & 0.68 & 3.82 & 1.06 & 0.74 & 4.79 & 1.06 & 0.84 & 5.51 \\
\hline \multirow{3}{*}{0.8} & 0 & 2.44 & -0.17 & 6.39 & 2.04 & 0.09 & 6.34 & 1.91 & 0.21 & 6.89 \\
\hline & 0.1 & 1.79 & 0.07 & 5.46 & 1.62 & 0.23 & 5.86 & 1.70 & 0.22 & 6.98 \\
\hline & 0.2 & 0.96 & 0.66 & 3.66 & 1.04 & 0.71 & 4.54 & 0.99 & 0.86 & 5.02 \\
\hline \multirow{3}{*}{0.7} & 0 & 2.35 & -0.14 & 6.03 & 2.03 & 0.09 & 6.14 & 1.72 & 0.34 & 6.00 \\
\hline & 0.1 & 1.78 & 0.07 & 5.33 & 1.59 & 0.23 & 5.64 & 1.48 & 0.37 & 6.02 \\
\hline & 0.2 & 0.90 & 0.69 & 3.41 & 0.95 & 0.76 & 4.17 & 0.89 & 0.93 & 4.48 \\
\hline \multirow{3}{*}{0.6} & 0 & 2.50 & -0.23 & 6.23 & 1.88 & 0.16 & 5.57 & 1.73 & 0.30 & 5.92 \\
\hline & 0.1 & 1.74 & 0.08 & 5.13 & 1.49 & 0.29 & 5.21 & 1.51 & 0.33 & 5.99 \\
\hline & 0.2 & 0.94 & 0.65 & 3.47 & 0.93 & 0.76 & 4.01 & 0.96 & 0.85 & 4.67 \\
\hline \multirow{3}{*}{0.5} & 0 & 3.01 & -0.54 & 7.22 & 1.72 & 0.22 & 5.01 & 1.58 & 0.36 & 5.27 \\
\hline & 0.1 & 1.69 & 0.09 & 4.92 & 1.54 & 0.24 & 5.22 & 1.32 & 0.44 & 5.22 \\
\hline & 0.2 & 0.89 & 0.66 & 3.28 & 0.91 & 0.75 & 3.86 & 0.91 & 0.85 & 4.40 \\
\hline \multirow{3}{*}{0.4} & 0 & 8.21 & -3.41 & 18.31 & 1.68 & 0.22 & 4.77 & 1.43 & 0.43 & 4.68 \\
\hline & 0.1 & 1.61 & 0.12 & 4.61 & 1.42 & 0.30 & 4.75 & 1.27 & 0.47 & 4.89 \\
\hline & 0.2 & 0.84 & 0.68 & 3.11 & 0.88 & 0.76 & 3.67 & 0.87 & 0.86 & 4.15 \\
\hline \multirow{3}{*}{0.3} & 0 & -29.6 & 17.00 & -61.8 & 1.63 & 0.22 & 4.48 & 1.34 & 0.46 & 4.25 \\
\hline & 0.1 & 1.61 & 0.10 & 4.53 & 1.30 & 0.36 & 4.31 & 1.28 & 0.44 & 4.77 \\
\hline & 0.2 & 0.81 & 0.68 & 2.95 & 0.85 & 0.76 & 3.50 & 0.82 & 0.88 & 3.87 \\
\hline
\end{tabular}


YAHYAOUI: Downwash Angle gradient

\begin{tabular}{|c|c|c|c|c|c|c|c|c|c|c|}
\hline \multirow{3}{*}{0.2} & 0 & -22.2 & 12.80 & -45.0 & 1.90 & 0.04 & 4.96 & 1.24 & 0.49 & 3.82 \\
\cline { 2 - 11 } & 0.1 & 1.67 & 0.048 & 4.57 & 1.31 & 0.34 & 4.20 & 1.17 & 0.49 & 4.30 \\
\cline { 2 - 11 } & 0.2 & 0.76 & 0.70 & 2.77 & 0.80 & 0.77 & 3.29 & 0.75 & 0.91 & 3.52 \\
\hline
\end{tabular}

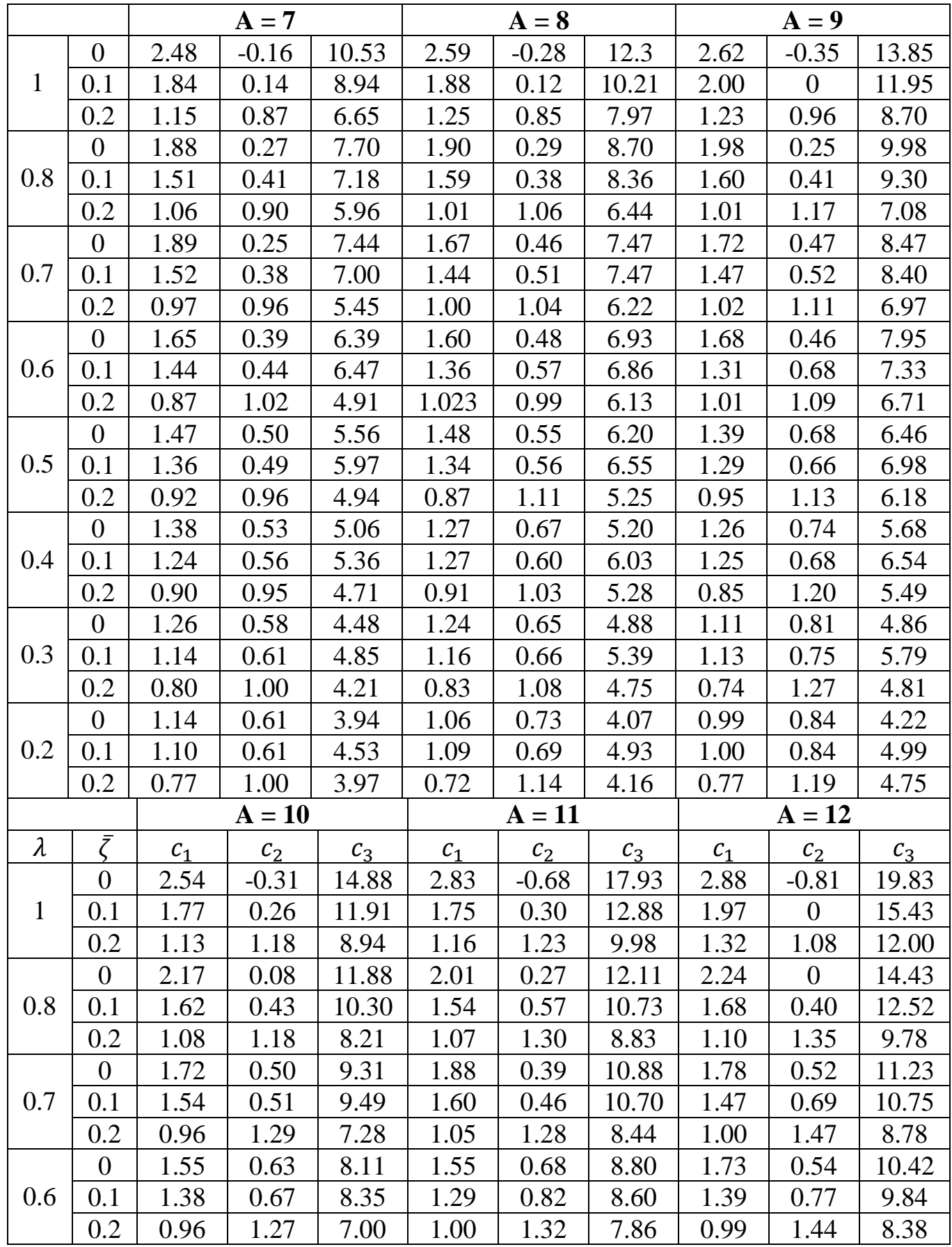




\begin{tabular}{|c|c|c|c|c|c|c|c|c|c|c|}
\hline \multirow{4}{*}{0.5} & 0 & 1.30 & 0.82 & 6.66 & 1.44 & 0.75 & 7.87 & 1.27 & 0.99 & 7.64 \\
\cline { 2 - 12 } & 0.1 & 1.43 & 0.57 & 8.28 & 1.31 & 0.76 & 8.35 & 1.41 & 0.70 & 9.52 \\
\cline { 2 - 11 } & 0.2 & 0.95 & 1.24 & 6.73 & 0.86 & 1.44 & 6.80 & 0.87 & 1.54 & 7.37 \\
\hline \multirow{3}{*}{0.4} & 0 & 1.27 & 0.80 & 6.20 & 1.29 & 0.86 & 6.76 & 1.16 & 1.03 & 6.71 \\
\cline { 2 - 11 } & 0.1 & 1.17 & 0.82 & 6.75 & 1.16 & 0.90 & 7.22 & 1.22 & 0.89 & 8.10 \\
\cline { 2 - 11 } & 0.2 & 0.86 & 1.29 & 6.05 & 0.89 & 1.36 & 6.69 & 0.86 & 1.48 & 7.07 \\
\hline \multirow{4}{*}{0.3} & 0 & 1.12 & 0.86 & 5.20 & 1.10 & 0.96 & 5.64 & 1.05 & 1.07 & 5.82 \\
\cline { 2 - 11 } & 0.1 & 1.12 & 0.82 & 6.12 & 1.04 & 0.99 & 6.31 & 1.02 & 1.07 & 6.68 \\
\cline { 2 - 11 } & 0.2 & 0.83 & 1.26 & 5.55 & 0.81 & 1.40 & 6.00 & 0.87 & 1.43 & 6.78 \\
\hline \multirow{3}{*}{0.2} & 0 & 1.02 & 0.90 & 4.66 & 0.99 & 0.99 & 4.89 & 0.97 & 1.08 & 5.11 \\
\cline { 2 - 11 } & 0.1 & 0.96 & 0.94 & 5.27 & 1.02 & 0.96 & 5.90 & 0.97 & 1.08 & 6.10 \\
\cline { 2 - 10 } & 0.2 & 0.73 & 1.34 & 4.96 & 0.72 & 1.45 & 5.29 & 0.74 & 1.53 & 5.77 \\
\hline
\end{tabular}

\section{The General Case of Swept and Tapered Wings}

The new empirical law for swept, tapered and untwisted wings is a generalization of the curve fitting approach previously applied to straight tapered wings except that constant $c_{2}$ is replaced by a function of wing sweep and constant $c_{3}$ is replaced by a function of the three main wing geometric parameters: aspect ratio, taper ratio, and sweep. The new law is of the form:

$$
\frac{\partial \varepsilon}{\partial \alpha}=\frac{1+c_{1} \bar{\xi}}{c_{2}^{\prime}+c_{3}^{\prime} \bar{\xi}}
$$

Where:

$$
\begin{aligned}
& c_{2}^{\prime}=c_{2}+1.8 \Lambda_{c / 4} \\
& c_{3}^{\prime}=c_{3}+\left[\left(C_{A \lambda}-C_{\lambda} \bar{\zeta}\right) \sin \Lambda_{c / 4}+\left(\sin \Lambda_{c / 4}\right)^{3.5}\right] \Lambda_{c / 4}
\end{aligned}
$$

The sweep angle $\Lambda_{c / 4}$ is expressed in radians and:

$$
\begin{aligned}
& C_{A \lambda}=1.1 A+12 \lambda-8.15 \\
& C_{\lambda}=35 \lambda-7.5
\end{aligned}
$$

Constants $\mathrm{c}_{1}, \mathrm{c}_{2}$, and $\mathrm{c}_{3}$ are the same constants given in Table 3 for an unswept, tapered and untwisted wing having the same aspect and taper ratios as the swept wing whose downwash angle gradient is to be determined. The main idea behind equation (10) was that the constant $c_{2}$ had to be increased since the curve representing $\partial \varepsilon / \partial \alpha$ tended to flatten out and rotate counter clockwise as the sweep angle was increased. Then the constant $c_{3}$ had to be increased in order to translate the curve vertically downwards and make it match as closely as possible the set of values obtained by the VLM. While the adjustment of constant $c_{2}$ was rather simple, that of constant $c_{3}$ was by no means obvious and was achieved through trial 
and error with some intuition and a great deal of perseverance. Computations were carried out for aspect ratios ranging from 4 to 10, taper ratios of 0.2 to 0.8 , and sweep angles of $20,25,30,35$, and $40^{\circ}$.

At $\bar{\zeta}=0$ and 0.1 , the maximum difference between the numerical values of $\partial \varepsilon / \partial \alpha$ and those given by equation (9) was less than $3 \%$. Figure 9 shows sample cases where the close agreement between the new empirical law and the numerical values is demonstrated. 

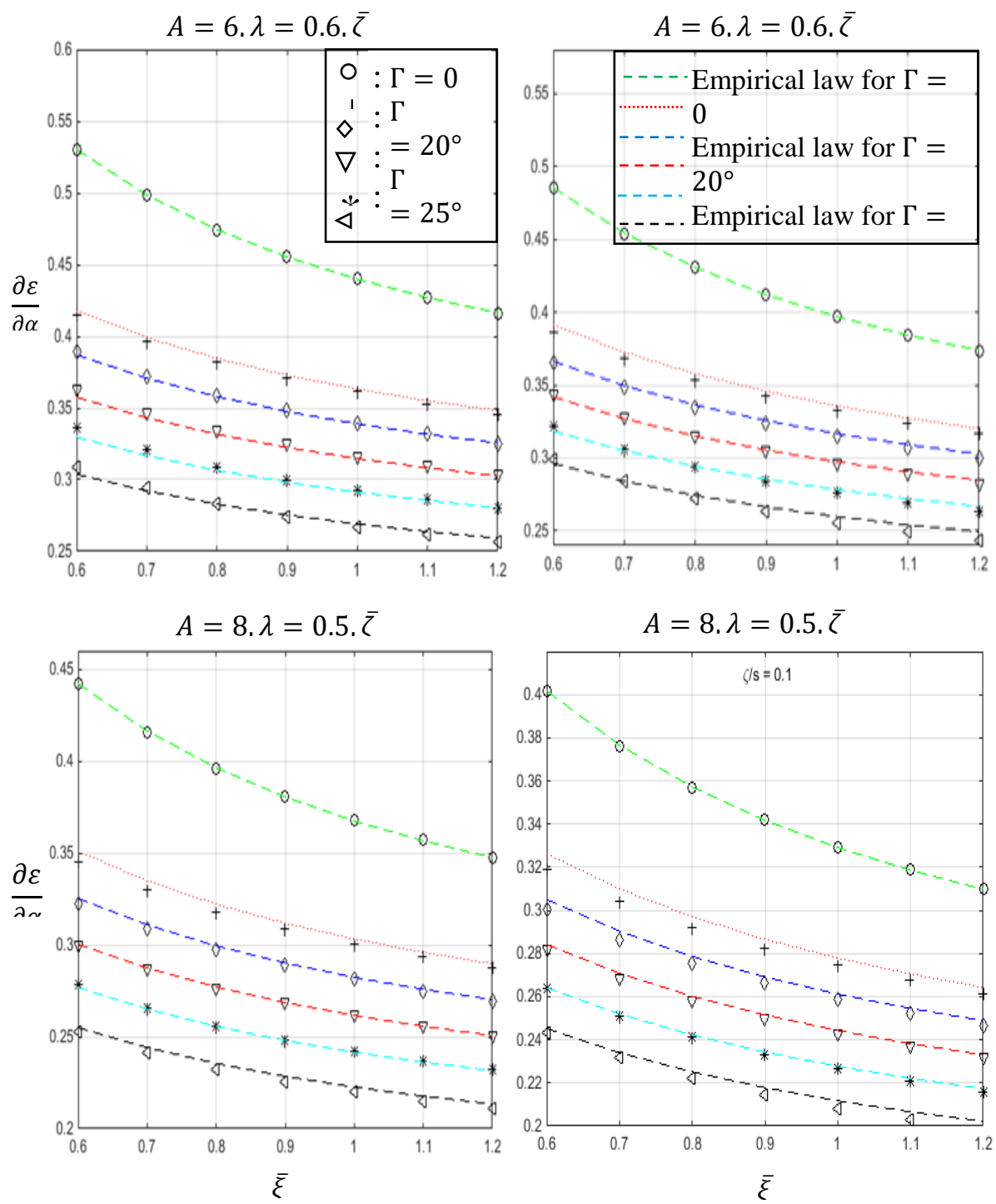

Figure 9. Sample cases of agreement between the new empirical law and VLM values of $\partial \varepsilon / \partial \alpha$ for given $\mathrm{A}, \lambda, \bar{\zeta}$, and different sweep angles.

\section{Example of Application}

For illustration purposes, the downwash angle gradient is computed for sample cases of untwisted straight and swept wings. The first case chosen is a wing 
of aspect ratio and taper ratio equal to 9 and 0.2 , respectively, and a horizontal tail which is located one half span behind the wing and zero distance above $(\bar{\xi}=1$ and $\bar{\zeta}=0$ ). For this case we have:

- The experimental value of $\partial \varepsilon_{c} / \partial \alpha$ at the centerline (Roskam, 1979) is 0.464 .

The average value is obtained by multiplying by a correction factor given in the same reference $\left(K=\varepsilon / \varepsilon_{c} \cong 0.9\right)$ to get $\partial \varepsilon / \partial \alpha=0.418$.

- The curve-fit method consists in getting constants $c_{1}, c_{2}$ and $c_{3}$ from Table 2 which are based on VLM computations:

$$
\mathrm{c}_{1}=0.99, \mathrm{c}_{2}=0.84 \text { and } \mathrm{c}_{3}=4.22
$$

then applying equation (7):

$$
\frac{\partial \varepsilon}{\partial \alpha}=\frac{1+c_{1} \bar{\xi}}{c_{2}+c_{3} \bar{\xi}}=\frac{1+0.99(1)}{0.84+4.22(1)}=0.393
$$

This differs from the experimental value by $-6 \%$.

- The empirical law from USAF-DATCOM (Hoak et al., 1978) is:

$$
\frac{\partial \varepsilon}{\partial \alpha}=4.44\left(K_{A} K_{\lambda} K_{H} \sqrt{\cos \Lambda_{c / 4}}\right)^{1.19}
$$

where:

$$
K_{A}=\frac{1}{A}-\frac{1}{1+A^{1.7}} \quad ; \quad K_{\lambda}=\frac{10-3 \lambda}{7} \quad ; \quad K_{H}=\frac{1-\zeta / b}{(2 \xi / b)^{1 / 3}}=\frac{1-\bar{\zeta} / 2}{\bar{\xi}^{1 / 3}}
$$

This law gives:

$$
\frac{\partial \varepsilon}{\partial \alpha}=0.349
$$

This differs from the experimental value by $-17 \%$.

Further comparison between experimental values, the USAF-DATCOM empirical law, and the present approach is given in Table 3. The cases considered were all for straight wings since no experimental data for swept wings was available. For the six cases considered, the average relative difference between the present approach and experimental values is $6.5 \%$ while the average difference between the USAF-DATCOM equation and experimental values is $12.3 \%$. 
Table 3

Comparison Between Experimental Data, the USAF-DATCOM Empirical Equation and the Present Approach for Values of the Downwash Angle Gradient (unswept wings)

\begin{tabular}{|l|c|c|c|c|c|c|}
\hline & $\mathrm{A}=6$ & $\mathrm{~A}=6$ & $\mathrm{~A}=6$ & $\mathrm{~A}=9$ & $\mathrm{~A}=9$ & $\mathrm{~A}=9$ \\
& $\lambda=1$ & $\lambda=1$ & $\lambda=0.2$ & $\lambda=1$ & $\lambda=1$ & $\lambda=0.2$ \\
& $\bar{\zeta}=0$ & $\bar{\zeta}=0.1$ & $\bar{\zeta}=0$ & $\bar{\zeta}=0$ & $\bar{\zeta}=0.1$ & $\bar{\zeta}=0$ \\
\hline Experim. & 0.426 & 0.385 & 0.514 & 0.293 & 0.268 & 0.419 \\
\hline VLM & 0.384 & 0.355 & 0.52 & 0.268 & 0.251 & 0.393 \\
& $(-10 \%)$ & $(-8 \%)$ & $(+1 \%)$ & $(-8 \%)$ & $(-6 \%)$ & $(-6 \%)$ \\
\hline USAF- & 0.361 & 0.339 & 0.512 & 0.2456 & 0.231 & 0.349 \\
DATCOM & $(-15 \%)$ & $(-12 \%)$ & $(0 \%)$ & $(-16 \%)$ & $(-14 \%)$ & $(-17 \%)$ \\
\hline
\end{tabular}

It is noted that the relative error between the present approach and experimental data has gone up from around 3\% for the downwash angle gradient at the centerline, to slightly over $6 \%$ when the comparison was based on the average downwash angle gradient. This is explained by the error brought in by the correction factor (Roskam, 1979) which was used to compute the average downwash angle gradient from the gradient at the center line.

The second case chosen is that of swept wing of aspect ratio and taper ratio equal to 8 and 0.5 , respectively, and a sweep angle at the quarter-chord line of $30^{\circ}$. The horizontal tail is located such that $\bar{\xi}=1$ and $\bar{\zeta}=0.1$. For this case:

- Our newly developed empirical law is applied as follows:

$$
\frac{\partial \varepsilon}{\partial \alpha}=\frac{1+c_{1} \bar{\xi}}{c_{2}^{\prime}+c_{3}^{\prime} \bar{\xi}}
$$

Constants $c_{1}, c_{2}$ and $c_{3}$ are obtained from Table 2 for an unswept wing of equal aspect ratio $(\mathrm{A}=8)$ and taper ratio $(\lambda=0.5)$. We get $\mathrm{c}_{1}=1.34, \mathrm{c}_{2}=0.56$ and $c_{3}=6.55$. Therefore:

$$
c_{2}^{\prime}=c_{2}+1.8 \Lambda_{c / 4}=0.56+1.8(\pi / 6)=1.50
$$

And:

$$
c_{3}^{\prime}=c_{3}+\left[\left(C_{A \lambda}-C_{\lambda} \bar{\zeta}\right) \sin \Lambda_{c / 4}+\left(\sin \Lambda_{c / 4}\right)^{3.5}\right] \Lambda_{c / 4}
$$

With:

$$
\begin{aligned}
& C_{A \lambda}=1.1 A+12 \lambda-8.15=6.65 \\
& C_{\lambda}=35 \lambda-7.5=10
\end{aligned}
$$

It follows that: 


$$
\begin{aligned}
& c_{3}^{\prime}=6.55+\left[(6.65-10 \times 0.1)(0.5)+(0.5)^{3.5}\right](\pi / 6)=8.08 \\
& \frac{\partial \varepsilon}{\partial \alpha}=\frac{1+(1.34)(1)}{1.50+(8.08)(1)}=0.24
\end{aligned}
$$

We note that the empirical law from USAF-DATCOM (Hoak et al., 1978) gives $\partial \varepsilon / \partial \alpha=0.3$. There is a relative difference of $20 \%$ with the value given by our empirical law.

\section{On the Effect of Wing Washout}

An attempt was made at including wing twist in the empirical law but the effort did not lead to any meaningful results. But it turns out, as shown in Figure 8 below, that this geometric parameter has limited effect on $\partial \varepsilon / \partial \alpha$.
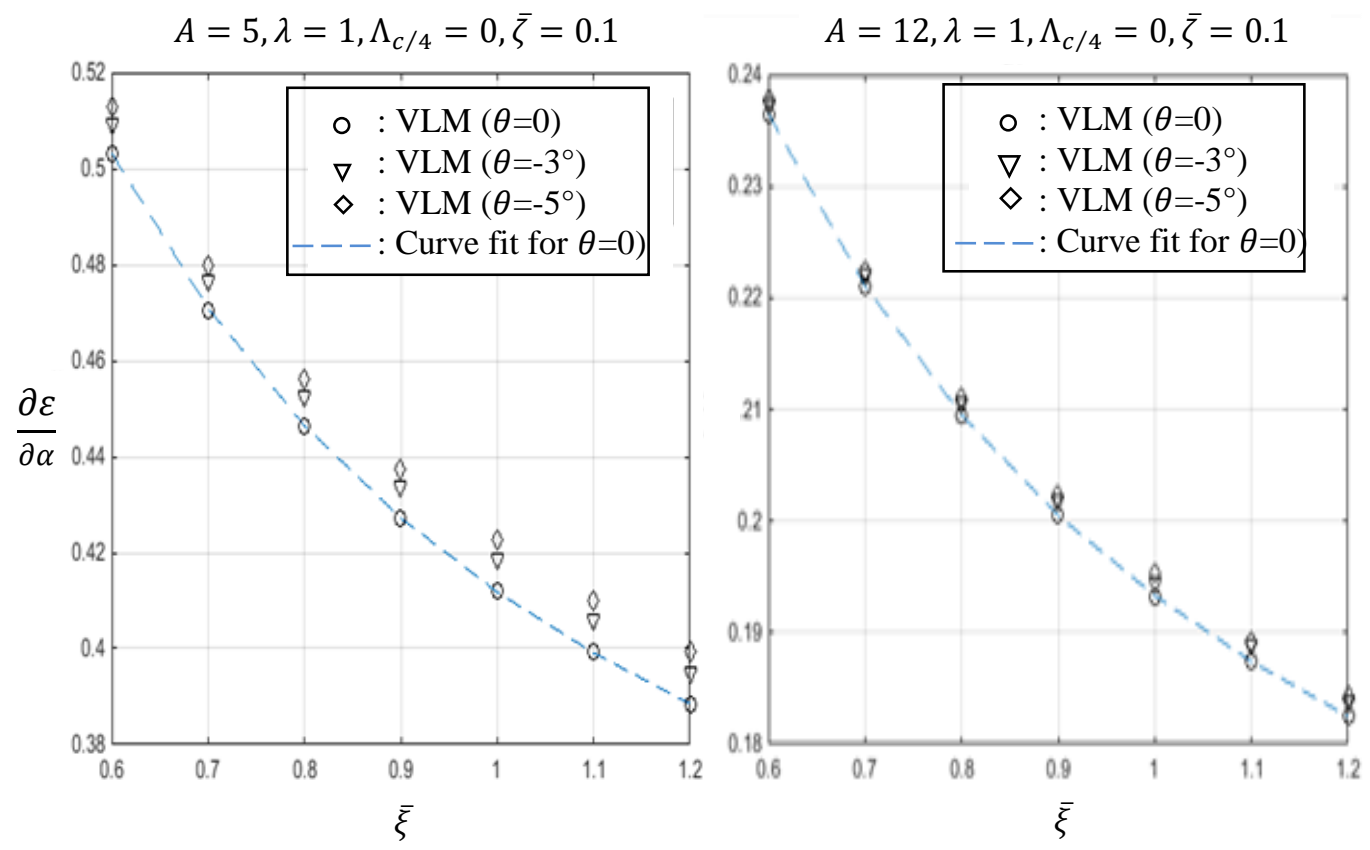

Figure 10. Effect of wing twist on $\partial \varepsilon / \partial \alpha$ for given values of $A$ and $\lambda$.

Comparing an untwisted rectangular wing to rectangular wing of equal aspect ratio and whose twist angle is $\theta=-5^{\circ}$, it is found that at a downstream distance $\bar{\xi}=1$, the relative increase in the downwash angle gradient for the twisted wing varies between $0.4 \%$ for $A=12$ and $\bar{\zeta}=0$ and $3 \%$ for $A=5$ and $\bar{\zeta}=0.2$. The general trend is that the increase in $\partial \varepsilon / \partial \alpha$ relative to the similar but untwisted wing is found to: 
- Decrease with aspect ratio: for rectangular wings with $-5^{\circ}$ twist, at a downstream distance $\bar{\xi}=1$ and at a vertical offset $\bar{\zeta}=0.1$ for instance, the increase in $\partial \varepsilon / \partial \alpha$ goes from $2.7 \%$ for $A=5$ down to $1 \%$ for $A=12$.

- Increase with the horizontal tail vertical offset $\bar{\zeta}$ : for a rectangular wing of aspect ratio 8 and $-5^{\circ}$ twist, at a downstream distance $\bar{\xi}=1$, the increase goes from $1 \%$ at $\bar{\zeta}=0$ to $1.9 \%$ at $\bar{\zeta}=0.2$.

- Vary very slightly with taper ratio: for wing of aspect ratio 8 and $-5^{\circ}$ twist, at a downstream distance $\bar{\xi}=1$, the increase goes from $1.6 \%$ for $\lambda=1$ to $1.8 \%$ for $\lambda=0.5$.

\section{Conclusion}

A new approach for the estimation of the downwash angle gradient aft of a straight rectangular or tapered wing in the incompressible regime has been introduced and it has been shown that this law can accurately duplicate numerical as well as experimental values of this important parameter. Then an empirical law for trapezoidal swept and tapered wings has been devised and has shown good accuracy when comparing the downwash angle gradient values with those generated numerically using the vortex-lattice method.

It has not been possible to include wing twist in the newly introduced empirical law but it has been demonstrated that this parameter has very limited effect on the downwash angle gradient. The increase in downwash angle gradient with wing twist is found to be around 3\% for wings with relatively low aspect ratio and a washout of $5^{\circ}$. This is for an aft tail location of one half wing span and the higher value of vertical position of $20 \%$ of the half span. The increase is even less important at higher aspect ratios and lower vertical positions of the horizontal tail. 


\section{References}

Bertin, J. J., \& Cummings, R.M. (2009). Aerodynamics for engineers (5th ed.). New York, NY: Pearson Prentice-Hall.

Etkin, B., \& Reid, L.D. (1996). Stability and control (3rd ed.). New York, NY: John Wiley and Sons.

Hoak, D. E. (1978). USAF stability and control DAT-COM. Clayton, MO: Global Engineering Documents.

Levy, D. W. (1992, January). Prediction of average downwash gradient for canard configurations. Paper presented at the Thirtieth AIAA Aerospace Sciences Meeting \& Exhibit, Reno, NV, USA.

Phillips, J. D., (1985). Downwash in the plane of symmetry of an elliptically loaded wing. NASA TP-2414. Retrieved from https://ntrs.nasa.gov/ search.jsp?R=19850007384

Purser, P. E., Spearman, M. L., \& Bates, W. R. (1947). Preliminary investigation at low speed of downwash characteristics of small-scale swept wings. NACA TN N ${ }^{\circ}$ 1378. Retrieved from https://apps.dtic.mil/dtic/tr/fulltext/ u2/b804048.pdf

Raymer, D. P. (2012). Aircraft design: A conceptual approach (5th ed.). Washington, D.C.: American Institute of Aeronautics and Astronautics.

Roskam, J. (1979). Airplane flight dynamics and automatic flight controls. Ottawa, KS: Roskam Aviation and Engineering.

Silverstein, A., \& Katzoff, S. (1939). Design charts for predicting downwash angles and wake characteristics behind plain and flapped wings. NACA Technical Report 648. Retrieved from https://ntrs.nasa.gov/search.jsp? $\mathrm{R}=19930091722$

Silverstein, A., Katzoff, S., \& Bullivant, W. K. (1939). Downwash and wake behind plain and flapped airfoils. NACA Report 651. Retrieved from https://ntrs.nasa.gov/search.jsp?R=19930091726 